\title{
THE GAMMA-RAY SPECTRUM OF CLASSICAL NOVAE
}

\author{
M. HERNANZ ${ }^{1}$, J. JOSÉ ${ }^{2}$, J. GÓMEZ ${ }^{1}$
}

1. Centre d'Estudis Avançats de Blanes (CSIC), Cami de Sta. Bàrbara $s / n, 17300$ Blanes (Girona), Spain

2. Dept. Física i Eng. Nuclear, EUPVG, UPC, Avda. Victor Balaguer $s / n, 08800$ Vilanova i la G. (Barcelona), Spain

\begin{abstract}
We report on preliminary hydrodynamic simulations of thermonuclear runaways in the hydrogen-rich envelopes of accreting white dwarfs, leading to nova outbursts. A one-dimensional, Lagrangian, implicit, hydrodynamic code (Kutter \& Sparks 1972) has been used to reproduce the gross features of a classical nova, from the onset of accretion, through the progress of the outburst up to the expansion stage, where significant mass loss takes place. Special interest is focused on the synthesis of ${ }^{22} \mathrm{Na}$ and ${ }^{26} \mathrm{Al}$, for their interest in $\gamma$-ray line astrophysics. The $\gamma$-ray spectrum is built by means of a Monte Carlo simulation.
\end{abstract}

\section{A nova outburst: from the onset of accretion to mass ejection}

The evolution of a $1.25 \mathrm{M}_{\odot}$ carbon-oxygen white dwarf star, accreting matter at a rate of $\dot{M}_{\text {acc }}=1.610^{-8} \mathrm{M}_{\odot} \mathrm{yr}^{-1}$ has been computed. The accreted matter is assumed to be enhanced in CNO nuclei $(X=0.46, Y=$ $0.13, Z=0.41$ ) in order to power a fast nova. Detailed nucleosynthesis is obtained by following the evolution of 60 nuclei, ranging from ${ }^{1} \mathrm{H}$ to ${ }^{28} \mathrm{Si}$, and linked through an updated network that includes more than $260 \mathrm{nu}-$ clear reactions. An envelope of $M_{\text {env }} \simeq 110^{-5} \mathrm{M}_{\odot}$ is accreted on top of the white dwarf star through a hydrostatic phase. As soon as the temperature at the bottom of the accreted envelope reaches $T_{\mathrm{b}} \simeq 410^{7} \mathrm{~K}$, convection settles and extends gradually towards the star's surface. A peak energy generation of $1.410^{15} \mathrm{erg} \mathrm{g}^{-1} \mathrm{~s}^{-1}$, followed by a peak temperature of $T_{\max } \simeq 1.910^{8} \mathrm{~K}$ are obtained, $529 \mathrm{yr}$ from the beginning of the computations. At that time, convection reaches the surface, allowing the transport of short-lived $\beta^{+}$-unstable nuclei $\left({ }^{13} \mathrm{~N},{ }^{14} \mathrm{O},{ }^{15} \mathrm{O},{ }^{17} \mathrm{~F}\right)$ to the cool, 303

A. Evans and J. H. Wood (eds.), Cataclysmic Variables and Related Objects, 303-304. ๑ 1996 Kluwer Academic Publishers. Printed in the Netherlands. 
outer layers. The ejection phase is powered by the delayed energy emission from the $\beta^{+}$-unstable nuclei. A mass $7.410^{-6} \mathrm{M}_{\odot}$ is ejected $(72 \%$ of the envelope's mass) with velocities ranging from $958 \ldots 4010 \mathrm{~km} \mathrm{~s}^{-1}$. The maximum visual and bolometric magnitudes attained during the outburst are $M_{\mathrm{vis}}=-6.4$ and $M_{\mathrm{bol}}=-9.4$. The abundances, by mass, of ${ }^{22} \mathrm{Na}$ and ${ }^{26} \mathrm{Al}$ synthesized are $6.310^{-4}$ and $9.810^{-3}$, respectively.

\section{Gamma-ray spectrum}

During the nova outburst, several unstable nuclei are synthesized with abundances high enough to produce a strong $\gamma$-ray emission in their decay (Livio et al. 1992, Starrfield et al. 1992). These nuclei are divided into (i) short lived positron emitters which take part in the CNO cycle $\left({ }^{13} \mathrm{~N}\right.$, ${ }^{14} \mathrm{O},{ }^{15} \mathrm{O}$ and ${ }^{17} \mathrm{~F}$ ) and (ii) ${ }^{26} \mathrm{Al}$ and ${ }^{22} \mathrm{Na}$ (Weiss \& Truran 1990). Only the emission produced by the decay of ${ }^{22} \mathrm{Na}$ might be detectable by COMPTEL or INTEGRAL. The ${ }^{22} \mathrm{Na}$ decays following the chain ${ }^{22} \mathrm{Na} \rightarrow{ }^{22} \mathrm{Ne}$, producing a well defined $\gamma$-ray line at $1270 \mathrm{keV}$, and positrons which annihilate emitting $511 \mathrm{keV}$ photon pairs. By means of a Monte Carlo code (Ambwani $\&$ Sutherland 1988), the spectra resulting from the interaction of these photons with the nova envelope have been calculated. The following physics is included in the code: (i) $\mathrm{e}^{-}-\mathrm{e}^{+}$pair production, (ii) photoelectric absorption, (iii) non-conservative Compton scattering, (iv) $\mathrm{e}^{-}-\mathrm{e}^{+}$annihilation, and $(\mathrm{v})$ positronium formation. The calculations have been carried out at different times starting at $2300 \mathrm{~s}$ after the onset of the outburst. In all cases, the spectra are obtained by using the channels defined by the expected resolution of INTEGRAL and also, for comparison purposes, the spectra have been convolved with the COMPTEL (CGRO) spectral response. At $250 \mathrm{pc}$ the continuum would not be detectable by COMPTEL, while INTEGRAL would detect part of it from several hours to 1 or $2 \mathrm{~d}$ after outburst. Concerning the $1270 \mathrm{keV}$ line, an intensity of $210^{-5}$ counts $\mathrm{cm}^{-2} \mathrm{~s}^{-1}$ would be obtained for our model for a nova at a distance of $1 \mathrm{kpc}$.

\section{References}

Ambiwani, K., Sutherland P., 1988, Ap. J., 325, 820

Kutter, G. S., Sparks, W. M., 1972, Ap. J., 175, 407

Livio, M., Mastichiadis, A., Ogelman, H., Truran, J. W., 1992, Ap. J., 394, 217

Starrfield, S., Shore, S. N., Sparks, W. M., et al., 1992, Ap. J., 391, L71

Weiss, A., Truran, J. W., 1990, A\&A, 238, 178 